\title{
Central ossifying fibroma: A case report
}

\author{
Mukesh Kumar ${ }^{1, *}$, Lakshami Gandhi², Ashish Kumar Rai ${ }^{3}$, Jitender Chaudhary $^{4}$ \\ ${ }^{1}$ Reader, ${ }^{2}$ Professor \& HOD, ${ }^{3,4}$ Post Graduate, Dept. of Oral Maxillofacial Surgery, Shree Bankey Bihari Dental College,
}

Ghaziabad, Uttar Pradesh, India

*Corresponding Author:

Email: kumardrmukesh@gmail.com

\begin{abstract}
Central ossifying fibroma is a relatively rare, benign, non-odontogenic tumor of the jaw, a subdivision of fibro-osseous lesions. The lesion originates from periodontal membrane and is usually seen in tooth bearing areas particularly of the mandible. This bone tumor consists of highly cellular, fibrous tissue that contains varying amounts of calcified tissue resembling bone, cementum or both. It is most commonly seen between the third and fourth decades of life. The most common location is the mandible. The lesion is generally asymptomatic until the growth produces a noticeable swelling and mild deformity. Displacement of teeth may be an early clinical feature. Here we present a case of cemento-ossifying fibroma of the jaw not only based on clinical characteristics but also radiographic and histopathologic features as an adjunct.
\end{abstract}

Keywords: Benign odontogenic tumour, Fibro-osseous lesions, Ossifying fibroma.

\section{Introduction}

Central ossifying fibroma is a relatively rare, benign, non-odontogenic tumor of the jaw, a subdivision of fibro-osseous lesions. The lesion originates from periodontal membrane and is usually seen in tooth bearing areas particularly of the mandible. ${ }^{1}$ This bone tumor consists of highly cellular, fibrous tissue that contains varying amounts of calcified tissue resembling bone, cementum or both. ${ }^{1,2}$ It is most commonly seen between the third and fourth decades of life. It is more frequent in women than in men (4:1). The most common location is the mandible. The lesion is generally asymptomatic until the growth produces a noticeable swelling and mild deformity. Displacement of teeth may be an early clinical feature. The most common location is in premolar-molar region of the mandible, with $62 \%$ to $89 \%$ of patients, $77 \%$ occurring in the premolar and 75 to $89 \%$ in molar region and in maxilla, $11 \%-26 \%$ of cases. ${ }^{3}$ Clinically, these tumors manifest as slow-growing intra-bony asymptomatic mass, over a period of time lesion may become large enough to cause facial asymmetry. ${ }^{1,4}$ Radiologically, central ossifying fibroma presents as a well-defined, unilocular lesion which contains varying amounts of radiopaque material. Once it is completely excised, central ossifying fibroma does not usually recur. ${ }^{5}$

\section{Case Report}

A 18 year old female patient reported to our department with mild pain and swelling in the left back teeth region for the past one week. The history of present illness revealed that the pain started one week back which was intermittent in nature. The patient reported that the swelling was present for the past 2 years. Since the swelling was not associated with pain all these years, patient had not taken any treatment for the same. On extra-oral examination, Revealed mild diffuse swelling in the left mandibular region extending from the commissures to the inferior border of the mandible in superio-inferior direction and anterior border of the ramus to the chin area in postero-anterior direction.[Fig. 1] On intraoral examination, there was missing 33. There was a firm swelling extending from mesial side of 32 up to 36 regions, covering the occlusal surface of 35 . There was obliteration of buccal and lingual cortical area in 32 and 36 regions. [Fig. 2a, and $\mathrm{b}$ ]

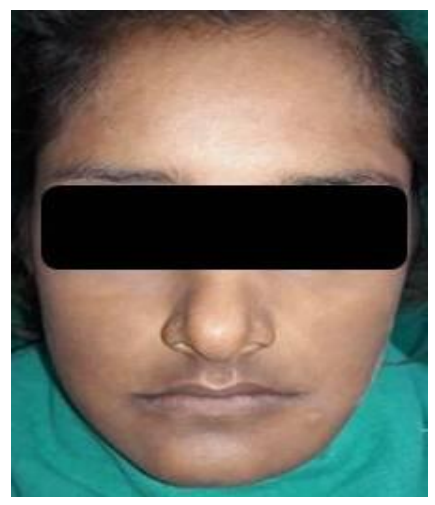

Fig. 1: Profile view showing mild asymmetry on left side of lower $1 / 3$ rd of face

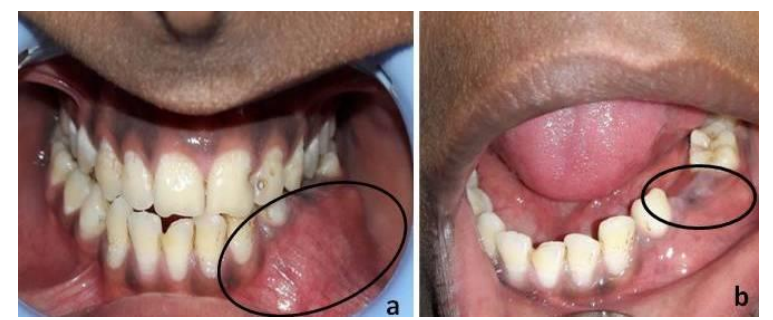

Fig. 2: Intra-oral view showing mild obliteration of left buccal vestibule (a) and missing 33 (b) 
Routine baseline investigations are noncontributory. On radiographic examination an orthopantamograph showed a well defined round unilocular radiolucency from the periapical region of 32 to 35 region mesiodistally and upto the inferior border of the mandible inferiorly. There were small areas of radiopacities seen inside the radiolucent region. [Fig. 3]

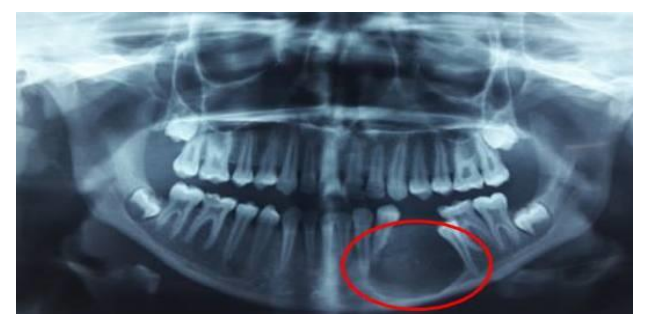

Fig. 3: Orthopantogram revealed a unilocular radiolucency with multiple tiny radiopacities

Based on clinical examination and radiolographical findings diagnosed as ossifying fibroma. Complete surgical excision of the lesion was done [Fig. 4] and sent for histopathological study which revealed mesenchymal tumour comprised of the thin wavy spindle cells arranged as fascicles and whorls with scattered spindle of dark blue osteoid material revealed nature of ossifying fibroma. [Fig. 5] Patient was reviewed post treatment follow-up after 6 months and found normal without any abnormalities. [Fig. 6,7]

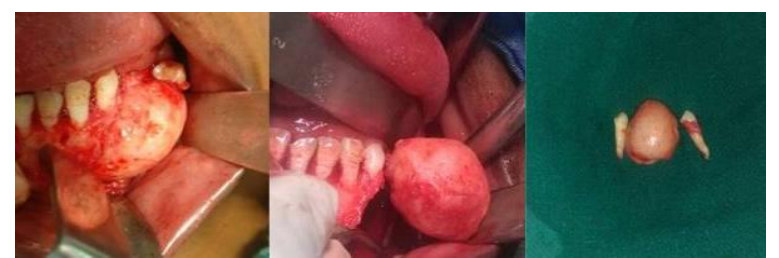

Fig. 4: Surgical procedure showing extracted tooth and excised lesions

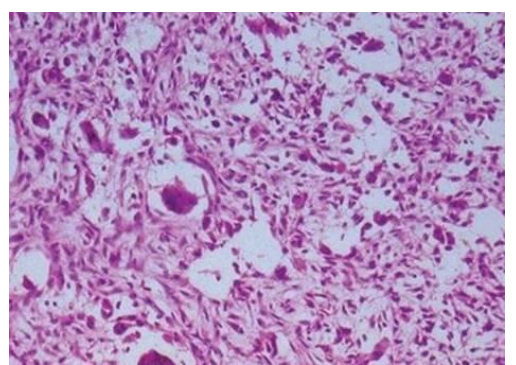

Fig. 5: Histopathology picture

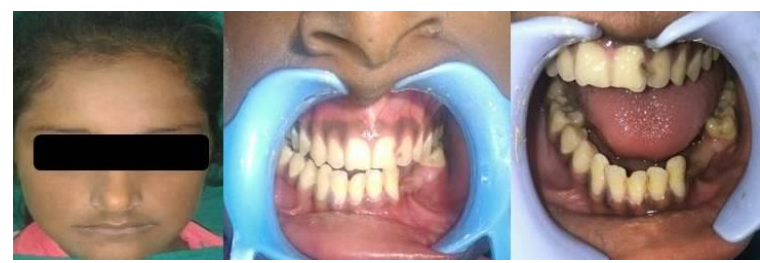

Fig. 6: Postoperative photographs after 6 months

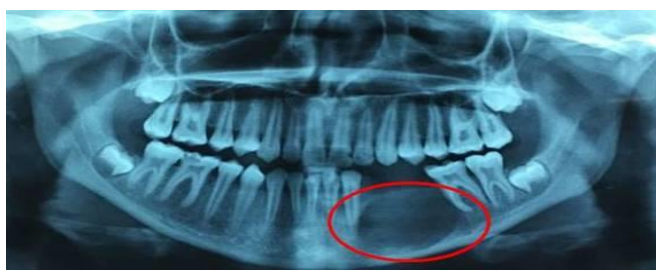

Fig. 7: Post-operative radiograph showing healed enucleated alveolar bony pattern

\section{Discussion}

The term 'ossifying fibroma' (OF) has been used since 1927, and since 1968 cementum-containing tumors have been grouped together. ${ }^{1}$ In 1971 the World Health Organization (WHO) classified four types of cementum-containing lesions: fibrous dysplasia, ossifying fibroma, cementifying fibroma and cementoossifying fibroma. ${ }^{2}$ According to the second WHO classification, benign fibro-osseous lesions in the oral and maxillofacial regions were divided into two categories, osteogenic neoplasm and non-neoplastic bone lesions; cementifying ossifying fibroma belonged to the former category. ${ }^{3}$ However, the term 'cementifying ossifying fibroma' was reduced to ossifying fibroma in the new WHO classification in $2005 .{ }^{4}$ The origin of OF is thought to be the periodontal membrane. ${ }^{5}$ Most cases of active OFs are asymptomatic, and the first clinical manifestation is a swelling of the mandibular cortical layer, which produces a marked extraoral facial asymmetry. ${ }^{6}$ The growth of the lesion may result in displacement of teeth or the inferior alveolar canal. A significant point is that the outer cortical plate, although displaced and thinned, remains intact. The lamina dura of involved teeth usually is missing. ${ }^{?}$

Radiographic features are non-specific and typically consist of a unilocular or multilocular radiolucency having ill-defined borders and occasional central opacification. Aggressive lesions may show cortical thinning and perforation and the lesions are either completely radiolucent or mixed, depending on the amount of calcification, or are completely radiopaque and surrounded by a radiolucent rim. In each type, there is a sclerotic border around the lesion. Multilocularity is rare. ${ }^{6}$ Lu et al., reported four radiographic patterns for $\mathrm{OF}$, namely, cystic radiolucency, ground glass appearance, sclerotic change, and mixed type, while Barberi et al., reported three radiographic patterns for $\mathrm{OF}$, namely, radiolucent $(53 \%)$, sclerotic $(7 \%)$, and mixed type $(40 \%){ }^{8}$

Histologically, OF is a well-demarcated lesion consisting of a fibroblastic stroma containing of plexiform and lamellar bone in addition to acellular mineralized material. ${ }^{8}$ Presence of hypercellular fibrous tissue with the occasional presence of islands of bone tissue or cementiform calcifications. Within the fibrous stroma, mineralized tissue masses corresponding to 
osteoid material or cement were seen accompanied by dystrophic calcifications. ${ }^{9}$ Juvenile trabecular fibroma (JTOF) and juvenile psammomatoid ossifying fibroma (JPOF) are other histological OF types. In contrast with conventional OF, JTOF and JPOF occur most commonly in the maxilla and bony walls of paranasal sinuses, respectively. Both present predilection for patients up to the second decade of life. JTOFs usually show an aggressive and rapid growth. ${ }^{10,11}$ Differential diagnosis includes osteoblastoma, an intraosseous form of fibromatosis known as desmoplastic fibroma, cementoblastoma, and osteoid osteoma. ${ }^{6}$ The treatment of ossifying fibroma is a complete removal by curettage or excision. ${ }^{12,13}$

\section{Conclusion}

The diagnosis of cemento-ossifying fibroma of the jaw can be established based on clinical, radiographic and histo-pathological features. It is a benign growth, considered to originate from the periodontal ligament and presents as a slow-growing lesion, but may cause deformity if left untreated. It is often encapsulated and predominantly located in the mandible, and the definitive diagnosis of these lesions requires integration of its clinical, radiological and histological features.

\section{References}

1. Hamner JE, Scofied HH, Cornyn J. Benign fibro-osseous jaw lesions of periodontal membrane origin. An analysis of 249 cases. Cancer 1968;26:861-78.

2. Pindborg JJ, Kramer IRH. Histological typing of odontogenic tumors, jaw cysts and allied lesions. In: International histological classification of tumors. Geneva: WHO, 1971, pp 31-4.

3. Kramer IRH, Pindborg JJ, Shear M. Neoplasm and other lesions related to bone. Histologic typing of odontogenic tumors, World Health Organization. Berlin, SpringerVerlag 1992:28-31.

4. Reichart PA, Philipsen HP, Sciubba JJ. The new classification of Head and Neck Tumours (WHO) - any changes? Oral Oncol 2006;42:757-8.

5. Liu Y, Wang H, You M, Yang Z, Miao J, Shimizutani K, et al. Ossifying fibromas of the jaw bone: 20 cases. Dentomaxillofac Radiol. 2010;39:57-63.

6. Toro C, Millesi W, Zerman N, Robiony M, Politi M. A case of aggressive ossifying fibroma with massive involvement of the mandible: Differential diagnosis and management options. Int J Pediatr Otorhinolaryngol. 2006;1:167-72.

7. Imanimoghaddam M, Hosseini Zarc SH, Nemati S, Javadian Langaroody A. Cemento-ossifying fibroma: Study of radiographic features of six cases. Iran J Radiol. 2009;6:215-20.

8. Barberi A, Cappabianca S, Collela G. Bilateral cementossifying fibroma of the maxillary sinus. $\mathrm{Br} \mathrm{J}$ Radiol. 2003;76:279-80.

9. Silvestre-Rangil J, Silvestre FJ, Requeni-Bernal J. Cemento-ossifying fibroma of the mandible: Presentation of a case and review of the literature. J Clin Exp Dent. 2011;3:66-9.

10. Slootweg PJ. Osseous dysplasia. In: Barnes L, Eveson JW, Reichart P, Sidransky D, eds. Pathology \& Genetics Head and Neck Tumors. Lyon: IARC Press; 2005. p.323.
11. Slootweg PJ. Lesions of the jaws. Histopathology 2009;54:401-18.

12. Chang CC, Hung HY, Chang JY, Yu CH, Wang YP, Liu $\mathrm{BY}$, Chiang CP. Central ossifying fibroma: a clinicopathologic study of 28 cases. J Formos Med Assoc 2008;107:288-94.

13. Triantafillidou K, Venetis G, Karakinaris G, Iordanidis F. Ossifying fibroma of the jaws: a clinical study of 14 cases and review of the literature. Oral Surg Oral Med Oral Pathol Oral Radiol 2012;114:193-9. 